\title{
USO DE BENZODIAZEPÍNICOS POR IDOSOS: sonolência diurna excessiva, instabilidade postural e adequação da prescrição na Estratégia de Saúde da Família
}

\author{
${ }^{1}$ Estudante do Curso de Graduação em $\quad$ Medicina da Associação $\quad$ Educativa do Brasil \\ (SOEBRAS).paulinhaalk@yahoo.com.br \\ ${ }^{2}$ Estudante do Curso de Graduação em Medicina da SOEBRAS.moniquedecarvalho21@ yahoo.com.br \\ ${ }^{3}$ Estudante do Curso de Graduação em Medicina da SOEBRAS.ludigodinho@ hotmail.com \\ ${ }^{4}$ Estudante do Curso de Graduação em Medicina da SOEBRAS.tam-veloso@ hotmail.com \\ ${ }^{5}$ Estudante do Curso de Graduação em Medicina da SOEBRAS. \\ ${ }^{6}$ Estudante do Curso de Graduação em Medicina da SOEBRAS.derlanmedicina2009@ gmail.com \\ ${ }^{7}$ Doutorando em Ciências da Saúde pela Universidade Estadual de Montes Claros (Unimontes). Docente do Instituto \\ Federal de Educação, Ciência e Tecnologia do Norte de Minas. Campus Pirapora.danilolimacarreiro@gmail.com \\ ${ }^{8}$ Mestre em Ciências da Saúde pela Unimontes. Docente da SOEBRAS. mineiro.laura@gmail.com \\ ${ }^{9}$ Mestre em Ciências da Saúde pela Unimontes. Docente da SOEBRAS.coutinhowlm@gmail.com
}

Recebido em: 09/01/2015 - Aprovado em: 15/06/2015 - Disponibilizado em: 15/07/2015

\begin{abstract}
RESUMO: Este estudo teve por objetivo investigar capacidade cognitiva, sonolência diurna excessiva, coordenação motora e instabilidade postural entre idosos usuários de benzodiazepínicos comparando-os com idosos não usuários e verificar adequação das prescrições deste psicotrópico por profissionais da Estratégia de Saúde da Família.Trata-se de umestudocomparativo entre idosos de três Unidades de Saúde de Montes Claros - MG. Dados coletados por pesquisadores calibrados utilizandoinstrumentos validados para uso no Brasil. Participaram 58 usuários e 232 não usuários deste psicotrópico. Registrou-seassociação entre uso de benzodiazepínico e: sonolência diurna excessiva $(\mathrm{p}=0,039)$ e instabilidade postural $(\mathrm{p}=0,011)$.A prescrição mostrou-se inadequada quanto: período de uso, dosagem e tipo de benzodiazepínicoprescrito.Os registros norteiam para a necessidade de ponderar os impactos do uso de benzodiazepínicos entre idosos, principalmente no que se refere à sonolência diurna excessiva e à instabilidade postural, bem como adequação dos profissionais da Estratégia de Saúde da Família quanto à prescrição deste fármaco.

Palavras-chave: Benzodiazepínicos. Idoso.Distúrbios do Sono por Sonolência Excessiva.Instabilidade Postural.Estratégia Saúde da Família.

\section{BENZODIAZEPINE USE BY ELDERLY: excessive daytime sleepiness, postural instability and appropriateness of prescribing in the Family Health Strategy}

\begin{abstract}
This study aimed to investigate cognitive ability, excessive daytime sleepiness, motor coordination and postural instability between benzodiazepine users elderly comparing them with older non-users and check appropriateness of prescriptions for psychotropic this professionals of the Family Health Strategy. This is a comparative study of elderly in three Health Services Montes Claros-MG. Data collected by researchers calibrated using validated instruments for use in Brazil. Participated 58 users and 232 nonusers this psychotropic. Registered association between use of benzodiazepine and: excessive daytime sleepiness $(p=0.039)$ and postural instability $(p=0.011)$. The prescription has proved inadequate as: period of use, dosage and type of prescribed benzodiazepine. Records guide to the need to consider the impacts of the use of benzodiazepines among elderly, particularly with regard to excessive daytime sleepiness and postural instability and appropriateness of health strategy for the professional family as the prescription of this drug.
\end{abstract}

Key words:Benzodiazepines.Elderly.Disorders of Excessive Somnolence.Postural instability.Family Health Strategy. 


\section{INTRODUÇÃO}

$\mathrm{O}$ aumento mundial do contingente populacional de idososé acompanhado pela modificação do perfil epidemiológico com incremento das condições crônicas. Realidade que reflete os avanços da medicina e aponta para a necessidade de mudanças e inovações nas políticas públicas de saúde, de modo que o idoso possa usufruir de forma saudável os anos proporcionados pelo avanço da ciência (BRASIL, 2006a; MENDES et al., 2012; PINHO et al., 2012).

Sujeitos idosos apresentam alteração dos padrões de absorção, distribuição e eliminação dos fármacos que podem gerar efeitos orgânicos tóxicos. Neste contexto merece destaque os benzodiazepínicos (BDZs), utilizados principalmentepara o tratamento da insônia e da ansiedade, transtornos mais prevalentes entre idosos. Todavia, sua prescriçãoquando feita de forma não racionalpode aumentar a morbimortalidadesendo necessário assim, cuidado especial em sua seleção, uso e aplicabilidade (AUCHEWSKI et al., 2004; BRASIL, 2006a; GORZONI et al., 2008; CAVADAS e RIBEIRO, 2011; FIRMINO et al., 2011; TELLES FILHO et al., 2011).

O tratamento da insônia secundária, distúrbio mais comum do sono na velhice, deve iniciar-se pelo tratamento da doença de base e o uso de BDZs somente será indicado no caso da insônia secundária transitória (inferior a quatro semanas de duração), após a tentativa de tratamento com medidas comportamentais, como a higiene do sono.A ansiedade por sua vez, não é primariamente uma doença, mas sim uma emoção necessária para o comportamento adequado do ser humano. Quando atinge um grau doentio, deve ser tratada utilizando a psicoterapia juntamente com o tratamento medicamentoso. Apesar de não constituem a droga de primeira linha para tratamento do transtorno de ansiedade, osBDZs são empregados largamente (AMB/CFM, 2008; NORDON e HÜBNER, 2009; CAVADAS e RIBEIRO, 2011;LEVITAN et al., 2011; QUINHONES e GOMES, 2011).

No Brasil, o atual modelo assistencial de saúde tem na Estratégia de Saúde da Família (ESF) o instrumento organizacional da atenção primária. Ao considerar tal serviço também como porta de entrada para assistência aproblemas psicológicos ou psicossociais, inclusive entre idosos, a prescrição indevida de psicotrópicos por clínicos gerais ou outros especialistas não psiquiatras e a acessibilidade facilitada a esta classe medicamentosa por programas governamentais e políticas de controle deficitárias pode refletir no uso inadequado deste fármaco (ORLANDI e NOTO, 2005; BRASIL 2006b; CRUZ et al., 2006; NORDON e HÜBNER, 2009; TELLES FILHO et al., 2011; SOUZA et al., 2012).

Neste sentido este estudo procurou investigar capacidade cognitiva, sonolência 
diurna excessiva, coordenação motora e instabilidade postural entre idosos cadastrados na ESF e usuários de BDZs, comparando-os com idosos não usuários deste fármaco e ainda verificar a adequação das prescrições deste psicotrópico pelos profissionais lotados neste nível de atenção.

\section{METODOLOGIA}

Estudo comparativo

entre idosos,usuários e não usuários de BDZs, cadastradosem três Unidades de Saúde da Família (USFs)em Montes Claros - MG. Os dados foramcoletados por pesquisadores examinadores previamente calibrados.

Consideraram-se como elegíveis idosos previamente diagnosticados pelos médicos das USFs com sintomas de insônia e/ou transtorno de ansiedade e que estivessem em uso de BDZs prescritos pelos médicos do serviço,independente do tempo de uso, sendo que estes compuseram o Grupo 1 (G1). Para efeito de comparação selecionaram-se quatro sujeitos entre seus vizinhos mais próximos, identificados arbitrariamente no sentido horário, que não fizessem uso de BDZs, pareados por sexo e idade, estes compuseram o Grupo 2 (G2). De modo a controlar o viés de seleção, considerou-se, uso da menor quantidade de medicamentos possíveis, uma vez que estes poderiam ser determinantes no desfecho. Não participaram do estudo pessoas acamadas, deficientes físicos e/ou mentais, dependentes e/ou usuários nocivos de substâncias psicoativas. Condições estas avaliadas a partir dos prontuários médicos das USFs. Ao se considerar tais critérios, por meio dos prontuários médicos das USFs, identificaram-se 58 usuários de BDZspara os quais foram selecionados 232 não usuários, totalizando 290 sujeitos. Destaca-se que todos os usuários de BDZsconsentiram em participar do estudo (taxa de resposta: 100\%). Para a coleta de dados utilizou-se protocolo constituído pelos seguintes instrumentos:Critérios de Beers-Fick, Mini Exame Mental (MEM), Escala de Sonolência de Epworth (ESE), Teste de Coordenação (COO), Time Up and Go Test (TUG), Critério de Classificação Econômica Brasil (CCEB) eQuestionário para avaliação de condições demográficas, socioeconômicas e de saúde.

-Critérios de Beers-Fick: utilizado para auxiliar o julgamento clínico na seleção dos medicamentos para idosos. Produzido a partir de parâmetros baseados em trabalhos publicados sobre medicamentos e farmacologia do envelhecimento, esse instrumento defini quais fármacos são potencialmente inapropriados para pessoas com 65 anos de idade ou mais. Dividem-se em: medicamentos ou classes medicamentosas que deveriam ser evitados em idosos, independentemente do diagnóstico ou da condição clínica devido o alto risco de efeitos colaterais e pela existência de outros fármacos mais seguros; e medicamentos ou classes medicamentosas que não devem ser 
usados em determinadas circunstâncias clínicas (GORZONI, FABBRI e PIRES, 2008; COSTA, 2009; OLIVEIRA et al., 2011). Informações sobre demais instrumentos são apresentados na Tabela 1.

Após identificar cada sujeito de G1 em seu respectivo domicílio, apresentava-se a metodologia da pesquisa aos mesmos e/ou aos seus responsáveis. Obtido o consentimento para participação no estudo, aplicavam-se na forma de entrevista os questionários e em seguida avaliava-se a coordenação motora e a estabilidade postural. Caso o idoso apresentasse dificuldades em responder aos instrumentos, solicitava-se auxílio ao seu cuidador. Para avaliar os Critérios de BeersFickconsideraram-seas prescrições do prontuário médico, bem como se solicitou ao idoso a apresentação dos medicamentos em uso e sua posologia.Após três visitas sem sucesso de encontrar os sujeitos de G2, estes eram desconsiderados do estudo, sendo alocado outro para substituí-lo.

Os dados foram analisados através do software Statistical Package for the Social Sciences (SPSS) v. 17.0. Para comparar grupos considerando as variáveis qualitativas, utilizou-se teste Qui-quadrado de Pearson $\left(\mathrm{X}^{2}\right)$. Para as variáveis quantitativas compararam-se os valores médios da idade, da segmentação econômica, da capacidade cognitiva, da instabilidade postural e da sonolência excessiva diurna pelo teste $\mathrm{T}$ de Student. Todos os testes consideraram o nível de significância de $\mathrm{p} \leq 0,05$.Por fim, foram conduzidas análises descritivas com o intuito de caracterizar a amostra e descrever somenteG1 quanto aos critérios de BeersFick.

Estudo autorizado pelo Comitê de Ética e Pesquisa da Associação Educativa do Brasil com parecer consubstanciado ${ }^{\circ} 134.633$.

\section{RESULTADOS E DISCUSSÃO}

Registraram-serespectivas médias, desvios-padrão, intervalos de confiança a de idades entre G1 e G2: 71,57 anos $( \pm 6,28$; IC95\%: 69,92-73,22; 61-83) e 71,37 anos $( \pm 6,55 ; \quad$ IC95\%: 70,52-72,22; 60-84).Na Tabela 1, os idosos são caracterizados quanto às variáveis em estudo. Através do teste Quiquadrado análise bivariada evidenciou não existência de associação entre uso de BDZs e as variáveis em estudo (Tabela 1). 
Tabela 1: Comparação entre idosos usuários e não usuários de benzodiazepínicos quanto às variáveis individuais (condições demográficas e socioeconômicas) e condições de saúde (capacidade cognitiva, coordenação motora, instabilidade postural e sonolência diurna excessiva), 2012, $(\mathrm{n}=290)$

\begin{tabular}{|c|c|c|c|c|c|c|c|}
\hline \multirow[t]{2}{*}{ VARIÁVEIS } & \multicolumn{2}{|c|}{ Geral } & \multicolumn{2}{|c|}{$\begin{array}{c}G 1^{*} \\
(\mathrm{n}=58)\end{array}$} & \multicolumn{2}{|c|}{$\begin{array}{c}G 2^{*} \\
(n=232)\end{array}$} & \multirow[b]{2}{*}{$p$} \\
\hline & $\mathbf{n}$ & $\%$ & $\mathbf{n}$ & $\%$ & $\mathbf{n}$ & $\%$ & \\
\hline \multicolumn{8}{|l|}{ Uso de benzodiazepínico } \\
\hline Não & 232 & 80,0 & - & - & - & - & - \\
\hline Sim & 58 & 20,0 & - & - & - & - & - \\
\hline \multicolumn{8}{|l|}{ INDIVIDUAIS } \\
\hline \multicolumn{8}{|l|}{ Demográficas } \\
\hline \multicolumn{8}{|l|}{ Sexo } \\
\hline Masculino & 71 & 24,5 & 15 & 21,1 & 56 & 78,9 & \\
\hline Feminino & 219 & 75,5 & 43 & 19,6 & 176 & 80,4 & 0,785 \\
\hline \multicolumn{8}{|l|}{ Cor ou raça $a^{a}$} \\
\hline Branca/amarela & 128 & 44,1 & 29 & 22,7 & 99 & 77,3 & \\
\hline Preta/parda & 162 & 55,9 & 29 & 17,9 & 133 & 82,1 & 0,315 \\
\hline \multicolumn{8}{|l|}{ Socioeconômicas } \\
\hline \multicolumn{8}{|l|}{ Estado civil $^{a}$} \\
\hline Casado & 157 & 54,1 & 28 & 17,8 & 129 & 82,2 & \\
\hline Divorciado/viúvo/desquitado & 120 & 41,4 & 27 & 22,5 & 93 & 77,5 & \\
\hline Solteiro & 13 & 4,5 & 3 & 23,1 & 10 & 76,9 & 0,605 \\
\hline \multicolumn{8}{|l|}{ Segmentação econômica ${ }^{b}$} \\
\hline Alta & 67 & 23,1 & 10 & 14,9 & 57 & 85,1 & \\
\hline Baixa & 223 & 76,9 & 48 & 21,5 & 175 & 78,5 & 0,236 \\
\hline \multicolumn{8}{|l|}{ Escolaridade } \\
\hline Oito ou mais & 79 & 27,2 & 11 & 13,9 & 68 & 86,1 & \\
\hline Um a sete anos & 211 & 72,8 & 47 & 22,3 & 164 & 77,7 & 0,117 \\
\hline \multicolumn{8}{|l|}{ Atividade laboral } \\
\hline Sim & 48 & 16,6 & 11 & 22,9 & 37 & 77,1 & \\
\hline Não & 242 & 83,4 & 47 & 19,4 & 195 & 80,6 & 0,581 \\
\hline \multicolumn{8}{|l|}{ CONDIÇÕES DE SAÚDE } \\
\hline \multicolumn{8}{|l|}{ Avaliação cognitiva $^{c}$} \\
\hline Melhor desempenho & 266 & 91,7 & 51 & 19,2 & 215 & 80,8 & \\
\hline Pior desempenho & 24 & 8,3 & 7 & 29,2 & 17 & 70,8 & 0,246 \\
\hline \multicolumn{8}{|l|}{ Coordenação motora $^{d}$} \\
\hline Bom / muito bom / regular & 211 & 72,8 & 38 & 18,0 & 173 & 82,0 & \\
\hline Fraco / muito fraco & 79 & 27,2 & 20 & 25,3 & 59 & 74,7 & 0,168 \\
\hline \multicolumn{8}{|l|}{ Instabilidade postural $^{e}$} \\
\hline Baixo & 287 & 99,0 & 58 & 20,2 & 229 & 79,8 & \\
\hline Moderado & 3 & 1,0 & 0 & 0,0 & 3 & 100 & -- \\
\hline Alto & 0 & 0,0 & -- & -- & -- & -- & -- \\
\hline \multicolumn{8}{|l|}{ Sonolência diurna excessiva ${ }^{f}$} \\
\hline Não & 272 & 93,8 & 54 & 19,9 & 218 & 80,1 & \\
\hline Sim & 18 & 6,2 & 4 & 22,2 & 14 & 77,8 & 0,808 \\
\hline
\end{tabular}

*G1 (usuários de benzodiazepínicos). G2 (não usuários de benzodiazepínicos).

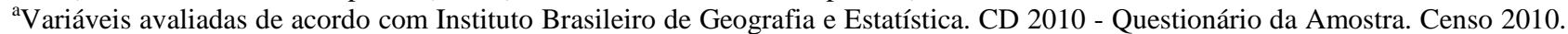
Disponível em: http://censo2010.ibge.gov.br/images/pdf/censo2010/questionarios/questionario_amostra_cd2010.pdf Acesso em: 09 jan. 2015.

${ }^{\mathrm{b}}$ Avaliada de acordo com o CCEB:desenvolvido pela Associação Brasileira de Empresas de Pesquisa (ABEP) tendo por finalidade estimar o poder de compra das pessoas e famílias urbanas. A classificação econômica considera as seguintes rendas familiares: A1 (R\$ 12.926,00); A2 (R\$ 8.418,00); B1 (R\$ 4.418,00); B2 (R\$ 2.565,00); C1 (R\$ 1.541,00); C2 (R\$ 1.024,00); D (R\$ 714,00) e E (R\$ 477,00)(FARIAet al., 2011). A segmentação deste estudo considerou: "Segmentação Econômica Alta" (pessoas com rendas familiares de: A1, A2, B1e B2); "Segmentação econômica Baixa" (pessoas com rendas familiares de: C1, C2, D e E) (RECHENCHOSKY et al., 2009).

${ }^{c}$ Avaliada de acordo com o Mini Exame Mental (MEM):validado para uso no Brasil (BRUCKIet al., 2003). Ferramenta de triagem de uso universal para avaliação cognitiva global, composto por questões relacionadas à orientação temporal e espacial, memória, atenção, habilidades de cálculo, linguagem e praxia construtiva. A pontuação varia de 0 a 30 , onde maior pontuação indica melhor desempenho cognitivo. Utilizou-se a versão modificada em relação às orientações temporal e espacial que se mostrou perfeitamente factível em estudos prévios. Adotou-se como pontos de cortes: 13 pontos (pessoas sem escolaridade); 18 (pessoas com um a sete anos de escolaridade) e 26 (pessoas com oito ou mais anos de escolaridade) (BRUCKIet al., 2003; CASTRO-COSTA et al., 2008; FARIAet al., 2011).

${ }^{d}$ Avaliada de acordo com o Teste de Coordenação (COO). Para tal, fixou-se um pedaço de fita adesiva com 76,2 cm de comprimento sobre uma mesa. Sobre a fita foram feitas seis marcas com $12,7 \mathrm{~cm}$ equidistantes entre si, com a primeira e a última marca a $6,35 \mathrm{~cm}$ de distância das extremidades da fita. Sobre cada uma das seis marcas fixou-se, perpendicularmente à fita, outro pedaço de fita adesiva com 7,6 cm de comprimento (OSNESS, 1990; ZAGO e GOBBI, 2003). O idoso foi orientado para sentar-se de frente para a mesa e usar sua mão dominante para realizar o teste. Se a mão dominante fosse a direita, uma lata de refrigerante seria colocada na 
posição "um", a lata "2" na posição "três" e, a lata "3" na posição "cinco". A mão direita era colocada na lata "1", com o polegar para cima, estando o cotovelo flexionado num ângulo de 100 a 120 graus. Ao sinal do pesquisador examinador, acionava-se o cronômetro e o idoso, virando a lata invertia sua base de apoio, de forma que a lata "1" fosse colocada na posição "dois"; a lata "2" na posição "quatro" e a lata "3" na posição "seis". Sem perder tempo, o idoso, estando com o polegar apontado para baixo, apanhava a lata "1" e invertia novamente sua base, recolocando-a na posição "um" e da mesma forma procedia colocando a lata " 2 " na posição "três" e a lata "3" na posição "cinco", completando assim um circuito. Uma tentativa equivalia à realização do circuito duas vezes, sem interrupções. Caso o idoso fosse sinistro, o mesmo procedimento foi adotado, exceto as latas que foram colocadas a partir da esquerda, invertendo-se as posições. A cada idoso foram concedidas duas tentativas, seguidas por outras duas válidas para avaliação, sendo estas últimas duas anotadas até décimos de segundo e, considerado como resultado final o menor dos tempos obtidos (ZAGO e GOBBI, 2003). O menor tempo obtido foi categorizado em escore percentil, e em seguida, categorizou-se em cinco partes iguais, obtendo cinco classificações: muito fraco (0-19); fraco (20-39); regular (40-59); bom (60-79) e muito bom (80-100) (ZAGO e GOBBI, 2003).

${ }^{e}$ Avaliada de acordo com Time Up and Go Test (TUG): avalia-se a estabilidade postural a partir do equilíbrio sentado, transferência de sentado para a posição de pé, estabilidade na deambulação e mudanças do curso da marcha, sem utilizar estratégias compensatórias. Para tal, solicitou-se ao idoso levantar-se de uma cadeira, deambular uma distância de três metros, virar-se, retornar e sentar-se na cadeira novamente, sendo o seu desempenho analisado em cada uma das tarefas através da contagem do tempo necessário para realizá-las. Considerou-se: baixo risco de quedas (tempo < 20 segundos); risco moderado (20-29 segundos); alto risco de quedas ( $\geq 30$ segundos) (GUIMARÃES et al., 2004; MACIEL e GUERRA, 2005; GAZZOLA et al., 2006; PRADO et al., 2010).

${ }^{\mathrm{f}}$ Escala de Sonolência de Epworth (ESE): validado para uso no Brasil (BERTOLAZI et al., 2009). Questionário autoaplicável que avalia a probabilidade de adormecer em oito situações envolvendo atividades diárias. Para graduar a probabilidade de cochilar, o indivíduo utiliza uma escala de "zero" a "três", onde "zero" corresponde à "nenhuma" e "três" à "grande probabilidade de cochilar". O escore global varia de "zero" a 24, sendo que os escores acima de 10 sugerem o diagnóstico de sonolência diurna excessiva (BOARI et al., 2004; BERTOLAZI et al., 2009).

\section{Entretanto, usando o teste T de Student}

para comparar as médias, encontrou-se

associação entre ser usuário e não ser usuário de benzodiazepínico e as variáveissonolência diurna excessiva $(\mathrm{p}=0,039)$ e instabilidade postural $(\mathrm{p}=0,011)($ Tabela 2$)$.

Tabela 2: Comparação de médias entre idosos usuários e não usuários de benzodiazepínicos quanto às variáveis individuais (condições demográficas e socioeconômicas) e condições de saúde (capacidade cognitiva, instabilidade postural e sonolência diurna excessiva), 2012, $(n=290)$

\begin{tabular}{|c|c|c|c|}
\hline \multirow[t]{2}{*}{ VARIÁVEIS } & \multicolumn{2}{|c|}{ Média } & \multirow[t]{2}{*}{$p$} \\
\hline & $\begin{array}{ll}G 1^{*} & \\
& (\mathrm{n}=58)\end{array}$ & $(n=232)$ & \\
\hline \multicolumn{4}{|l|}{ INDIVIDUAIS } \\
\hline \multicolumn{4}{|l|}{ Demográficas } \\
\hline Idade & 71,57 & 71,37 & 0,836 \\
\hline \multicolumn{4}{|l|}{ Socioeconômicas } \\
\hline Segmentação econômica & 18,37 & 19,09 & 0,386 \\
\hline \multicolumn{4}{|l|}{ CONDIÇÕES DE SAÚDE } \\
\hline Capacidade cognitiva & 24,52 & 25,23 & 0,167 \\
\hline Instabilidade postural & 10,56 & 9,32 & $0,011^{* * *}$ \\
\hline Sonolência diurna excessiva & 5,33 & 4,33 & $0,039^{* * *}$ \\
\hline
\end{tabular}

*G1 (usuários de benzodiazepínicos). G2 (não usuários de benzodiazepínicos).

*** value: $\leq 0,05$.

Utilizou-se o teste de Kolmogorov-Smirnov para verificar distribuição de normalidade dos dados e exequibilidade do teste T.

Todos os sujeitos de G1 (n=58) utilizam

BDZsde forma não racional, uma vez que

fazem uso contínuo da droga por período

médio bastante superior a quatro meses $(93,2$

meses); $15,51 \% \quad(\mathrm{n}=9)$ utilizam $\quad$ BDZs

inapropriados para idosos (oito idosos fazem 20mg/dia; e um idoso utiliza flurazepam na dosagem de 15mg/dia). Outros dois idosos, utilizam o hipnótico alprazolam com dosagens de $0,5 \mathrm{mg} / \mathrm{dia}$ e $2,0 \mathrm{mg} / \mathrm{dia}$, sendo considerado deletério para idoso doses superiores a $2,0 \mathrm{mg} / \mathrm{dia}$.

uso de diazepam nas dosagens de 10mg e/ou 
A associação entre uso de BDZs e instabilidade postural reforça a literatura especializada no assunto que orienta para se evitar o uso desta droga entre idosos, uma vez que entre seus efeitos colaterais se encontra a capacidade de provocar quedas com consequentes fraturas (BRASIL, 2006a;CARREIRO et al., 2010; COUTINHO et al., 2011; MENDES et al., 2012).Metaanálise que avaliou o papel de medicamentos psiquiátricos, cardiológicos e analgésicos sobre o risco de quedas entre idosos identificou que BDZs, neurolépticos, sedativos/hipnóticos, antidepressivos, diuréticos em geral, antiarrítmicos e digoxina associaram-se ao maior risco de quedas na população acima de 60 anos (LEIPZIG, CUMMING e TINETTI, 1999a; LEIPZIG, CUMMING e TINETTI, 1999b).Estudo casocontrole (idosos internados por uso de medicamentos e quedas seguidas de fraturas graves; e idosos cuja doença que determinou a internação não estava associada ao uso dos medicamentos) observou aumento no risco desses acidentes entre pessoas em uso de BDZs (OR=2,09, 1,08-4,05) (COUTINHO e SILVA, 2002). Tal associação pode explicarse por propriedades medicamentosas dos BDZs como, alterações psicomotoras por atividade sedativa e aumento da probabilidade dehipotensão postural por bloqueio adrenérgico do fármaco (RAY et al., 1987).

A associação entre uso de BDZse ocorrência de sonolência diurna excessiva constatada neste trabalho é citada em outros estudos como efeito adverso comum decorrente do uso desta classe de medicamento, inclusive no Projeto Diretrizes da Sociedade Brasileira de Psiquiatria (AUCHEWSK et al., 2004; BRASIL, 2006a; NORDON e HÜBNER, 2009;CAVADAS e RIBEIRO, 2011;TELLES FILHO et al., 2011; MENDES et al., 2012).De origem multifatorial, a sonolência diurna excessiva, é um sintoma comum entre idosos, com prevalência e intensidade que aumentam com a idade, tendo dentre seus fatores causais, o uso indevido e/ou abusivo de fármacos esendo considerada fator de risco para acidentes, problemasinterpessoais e impacto negativo na produtividade e na percepção do nível de qualidade de vida. Desta forma, é fundamental que previamenteà prescrição e administração de BDZs para pessoas idosas, considerem-se os efeitos colaterais e indesejáveis desta classe, bem como sua interação com as demais drogas que, em alguns casos, podem ser até mesmo fatais (SOUZA, MAGNA e PAULA, 2003).

Averiguou-se que $100 \%$ dos usuários de BDZs extrapolaram o período de consumo indicado para o uso do medicamento, ou seja, quatro meses. Diversos estudos demonstraram o uso inadequado dessa classe farmacológica, como consumo por tempo prolongado e utilização para alívio de quadros inespecíficos. Atualmente, esses medicamentos são indicados apenas para o 
tratamento agudo e subagudo de ansiedade, insônia e crises convulsivas, sendo injustificável sua utilização por mais de quatro meses quando há a diminuição do seu efeito ansiolítico, maior risco de tolerância, insônia rebote e dependência (AMB/CFM, 2008; NORDON e HÜBNER, 2009; NORDON et al., 2009; CAVADAS e RIBEIRO, 2011; FIRMINO et al.., 2011; SOUZA, OPALEYE e NOTO, 2013). Ressalta-se que mesmo mediante divulgações científicas acerca dos riscos da utilização prolongada deste fármaco, usuários cadastrados em programas de saúde, são os que permanecem como maiores consumidores desta classe medicamentosa, o que norteia para uma possível incapacidade de tais programas em abordar de forma adequada o uso racional deste medicamento (FIRMINO et al., 2011).

Constatou-se ainda que $15,51 \%$ dos usuários de BDZsfazem uso inapropriado ao se considerar os critérios de Beers-Fick, no qual o diazepam foi o medicamento mais frequentemente prescrito. Resultado este que corrobora com estudo que avaliou os fatores associados à prescrição de BDZs para os usuários do Serviço Municipal de Saúde de Coronel Fabriciano - MG, no qual a prevalência da prescrição de Diazepam $10 \mathrm{mg}$ correspondeu à $59,7 \%$ das notificações(FIRMINO et al., 2011).A frequente prescrição deste BDZ, potencialmente inapropriado para idosos, pode ser devido ao desconhecimento da farmacodinâmica e fisiologia na senescência por parte dos médicos; à indisponibilidade temporal dos profissionais para orientar os pacientes em relação a terapêuticas alternativas para melhora das queixas de insônia e ansiedade; à tentativa de manter uma boa relação médico-paciente; à subestimação da gravidade do uso, dos efeitos colaterais; à não observação das guidelines e ao fato desta classe medicamentosa compor a lista de fármacos para a atenção básica com financiamento integral pelo setor público (SOUZA, MAGNA e PAULA, 2003; AUCHEWSKI et al., 2004; COSTA, 2009; FIRMINO et al., 2011; NORDON e HÜBNER, 2009; OLIVEIRA et al., 2011; TELLES FILHO et al., 2011).

O presente estudo tem como limitação o número de usuários de BDZs, o que pode ter implicado na impossibilidade de realizar análise estatística multivariada para melhor compreensão da associação entre o uso deste fármaco e variáveis independentes. Salientase que o processo que relaciona a avaliação das variáveis investigadas é dinâmico. Portanto, causas e efeitos certamente variam ao longo do tempo e, sendo este um estudo seccional, não é possível estabelecer uma relação temporal entre as associações observadas. Todavia, destaca-se que os resultados apresentados são confiáveis, frutos de um estudo epidemiológico o qual 
considerou os princípios básicos de controle dos vieses de seleção, aferição e confusão.

\section{CONCLUSÃO}

Os registros norteiam para a necessidade de ponderar os impactos do uso de benzodiazepínicos entre idosos, principalmente no que se refere à sonolência diurna excessiva e à instabilidade postural, bem como adequação dos profissionais da Estratégia de Saúde da Família quanto à prescrição deste fármaco.

\section{REFERÊNCIAS}

ABEP. Associação Brasileira de Empresas de Pesquisa. Critério de Classificação

Econômica Brasil. São Paulo; 2012.Disponível em <http://www.abep.org/new/> Acesso em 09 de janeiro de 2015.

AMB/CFM. Associação Médica Brasileira e Conselho Federal de Medicina. Projeto Diretrizes: transtorno de ansiedade: diagnóstico e tratamento. São Paulo; 2008.

Auchewski Let al. Avaliação da orientação médica sobre os efeitos colaterais de benzodiazepínicos. Rev Bras Psiquiatr. São Paulo, v. 26, n. 1, p. 24-31, 2004.

Bertolazi NA et al. Validação da escala de sonolência de Epworth em português para uso no Brasil. J Bras Pneumol.Brasília, v. 35, n. 9, p. 877-883, 2009.

Boari Let al. Avaliação da escala de Epworth em pacientes com a Síndrome da apneia e hiponímia obstrutiva do sono. Rev Bras Otorrinolaringol.São Paulo, v. 70, n. 6, p. 752-756, 2004.

Brasil. Ministério da Saúde. Caderno de Atenção Básica: envelhecimento e saúde da pessoa idosa. Brasília: Ministério da Saúde; 2006a.

Brasil. Ministério da Saúde. Cadernos de Atenção Básica à Saúde - no 19. Brasília: Ministério da Saúde; 2006b.

Brucki SMD et al. Sugestões para o uso do mini exame do estado mental no Brasil. Arq Neuropsiquiatr. São Paulo, v. 61, n. 3B, p. 777-781, 2003.

Carreiro DL et al. Quedas entre idosos: medidas profiláticas de fatores de riscos prescritas pela Estratégia de Saúde da Família. Rev Lecturas, Educación Física y Deportes,Buenos Aires, v. 15, n. 149, 2010.

Castro-Costa E et al. Normas para o exame do estado mini mental: ajuste do ponto de corte em estudos populacionais (evidências do estudo de saúde de Bambuí envelhecimento). Arq Neuro Psiquiatr.São Paulo, v. 66, n. $3^{\text {a }}$, p. 524-528, 2008.

Cavadas LF, Ribeiro L. Abordagem da insônia secundária do adulto nos cuidados de saúde primários. Acta Med Port.Lisboa, v. 24, n. 1, p. 135-144, 2011.

Costa SC. Avaliação da prescrição de medicamentos para idosos internados em serviço de clínica médica do Sistema Único de Saúde em um Hospital Público Universitário Brasileiro. 112 f. 2009. [Dissertação]. Belo Horizonte (MG): Universidade Federal de Minas Gerais; 2009.

Coutinho ESF, Silva SD. Uso de medicamentos como fator de risco para fratura grave decorrente de queda em idosos. Cad. Saúde Pública, Rio de Janeiro, v. 18, n. 5, p. 1359-66, 2002.

Coutinho WLM et al. Idosos adscritos na estratégia de saúde da família e seus conhecimentos referentes à prevenção de quedas. Rev Lecturas, Educación Física y Deportes, Buenos Aires, v. 16, n. 157, 2011.

Cruz AV et al. Uso crônico de diazepam em idosos atendidos na rede pública em Tatuí-SP. 
Rev Cienc Farm Basica Apl.Araraquara, v. 27, n. 3, p. 259-67, 2006.

Faria EC et al. Avaliação cognitiva de pessoas idosas cadastradas na estratégia saúde da família: município do Sul de Minas. Rev Esc Enferm USP.São Paulo, v. 45, Suppl: 2, p. 1748-52, 2011.

Firmino KF et al. Fatores associados ao uso de benzodiazepínicos no serviço municipal de saúde da cidade de Coronel Fabriciano, Minas Gerais, Brasil. Cad. Saúde Pública, Rio de Janeiro, v. 27, n. 6, p. 1223-32, 2011.

Gazzola JM et al. Fatores associados ao equilíbrio funcional em idosos com disfunção vestibular crônica. Rev Bras

Otorrinolaringol. São Paulo, v. 72, n. 5, p. 683-90, 2006.

Gorzoni ML, Fabbri RMA, Pires SL. Critérios de beers-fick e medicamentos genéricos no Brasil. Rev Assoc Med Bras.São Paulo9, v. 54, n. 4, p. 353-6, 2008.

Guimarães LHCT et al. Comparação da propensão de quedas entre idosos que praticam atividade física e idosos sedentários. Rev Neurociências, São Paulo, v. 12, n. 2, p. 69-73, 2004.

Leipzig RM, Cumming RG, Tinetti ME. Drugs and falls in older people: a systematic review and meta-analysis: I. Psychotropic drugs. J Am Geriatr Soc.v. 47, n. 1, p. 30-9, 1999a.

Leipzig RM, Cumming RG, Tinetti ME. Drugs and falls in older people: a systematic review and meta-analysis: II. Cardiac and analgesic drugs. J Am Geriatr Soc.47, n. 1, p. 40-50, 1999b.

Levitan MN et al. Diretrizes da Associação Médica Brasileira para o tratamento do transtorno de ansiedade social. Rev Bras Psiquiatr.São Paulo, v. 33, n. 3, p. 292-302, 2011.

Maciel ACC, Guerra RO. Fatores Associados à alteração da mobilidade em idosos residentes na comunidade. Rev Bras

Fisioter.São Carlos, v. 9, n. 1, p. 17-23, 2005.

Mendes ACG et al. Assistência pública de saúde no contexto da transição demográfica brasileira: exigências atuais e futuras. Cad Saúde Pública.Rio de Janeiro, v. 28, n. 5, p. 955-64, 2012.

Nordon DG, Hübner CK. Prescrição de benzodiazepínicos por clínicos gerais. Diagn Tratamento. São Paulo, v. 14, n. 2, p. 66-9, 2009.

Nordon DG et al. Características do uso de benzodiazepínicos por mulheres que buscavam tratamento na atenção primária.

Rev Psiquiatr.São Paulo, v. 31, n. 3, p. 1528, 2009.

Oliveira MG et al. Acesso a medicamentos potencialmente inapropriados em idosos no Brasil. Rev APS.Brasília, v. 14, n. 3, p. 25865, 2011.

Orlandi P, Noto AR. Uso indevido de benzodiazepínicos: um estudo com informantes-chave no município de São Paulo. Rev Latino-am Enfermagem. Ribeirão Preto, v. 13, Suppl: 1, p. 896-902, 2005.

Osness WH. Functional fitness assessment for adults over 60 years.Reston: American Alliance for Health, Physical Education, Recreation and Dance; 1990.

Prado RA et al. A influência dos exercícios resistidos no equilíbrio, mobilidade funcional e na qualidade de vida de idosas. O Mundo da Saúde.São Paulo, v. 34, n. 2, p. 183-91, 2010.

Pinho TAM et al. Avaliação do risco de quedas em idosos atendidos em Unidade Básica de Saúde. Rev Esc Enferm USP. São Paulo, v. 46, n. 2, p. 320-7, 2012.

Quinhones MS, Gomes MM. Sono no envelhecimento normal e patológico: aspectos clínicos e fisiopatológicos. Rev Bras 
Neurol.Rio de Janeiro, v. 47, n. 1, p. 31-42, 2011.

Ray WA et al. Psychotropic drug use and the risk of hip fracture. N Engl J Med.v. 316, n. 7, p. 363-9, 1987.

Rechenchosky Let al. Estado nutricional e perfil lipídico de crianças. Rev Educ

Física/UEM. Maringá, v. 20, n. 3, p. 431-40, 2009.

Souza ARL, Opaleye ES, Noto AR. Contextos e padrões do uso indevido de benzodiazepínicos entre mulheres. Ciênc Saúde Coletiva.Rio de Janeiro, v. 18, n. 4, p. 1131-40, 2013.

Souza JC, Magna LA, Paula TH. Sonolência excessiva diurna e uso de hipnóticos em idosos. Rev Psiq Clín.São Paulo, v. 30, n. 3, p. 80-5, 2003.

Souza SPO et al. Conhecimento sobre doenças e agravos de notificação compulsória entre profissionais da Estratégia Saúde da Família no município de Teresina, estado do Piauí, Brasil - 2010. Epidemiol. Serv. Saúde, Brasília, v. 21, n. 3, p. 465-74, 2012.

Telles Filho PCP et al. Utilização de benzodiazepínicos por idosos de uma estratégia de saúde da família: implicações para enfermagem. Esc Anna Nery.Rio de Janeiro, v. 15, n. 3, p. 581-6, 2011.

Zago AS, Gobbi S. Valores normativos da aptidão funcional de mulheres de 60 a 70 anos. Rev Bras Cienc Mov. Brasília, v. 11, n. 2, p. 77-86, 2003. 